\title{
Review of: "Composition of Mosquito Fauna and Insecticide Resistance Status of Anopheles gambiae complex in Itang special woreda, Gambella, Southwestern Ethiopia"
}

\author{
DANIEL NZIOKA MUNYWOKI
}

Potential competing interests: The author(s) declared that no potential competing interests exist.

This is a very important study with sound methodology. However, the following are some inputs for consideration.

\section{Abstract (Results section)}

The only member of An. gambiae s.I. identified in your study is An. arabiensis. Therefore, i suggest the authors should narrow down their results to this species only and NOT An. gambiae s.I.

The number of mosquitoes collected were 468 out of which 294 blood fed, 46 half gravid and gravid. Kindly account for the 128 not mentioned in the abstract.

\section{Background}

In 1st paragraph sentence two should be rewritten to have a clear meaning. Suggestion, insert "is" between parasite and transmitted.

In paragraph 7, formatting of kdr L1014F and L1014S should be consistent and conform to the rest in the paper.

\section{Anopheles mosquito larvae collection}

Restructure sentence 3 (Line 3) to have a clear meaning.

\section{Adult Anopheles mosquito collection}

The abbreviation PSC should be put in brackets on first mention or separate it with its full meaning with a comma.

\section{Insecticide susceptibility tests}

What criteria was used to choose the five insecticides used in this study. The reviewer suggests you include some general information on IRS in southwestern Ethiopia and the chemical insecticides in use to 
beef up your choice.

The last sentence in this section should have come earlier in the paragraph, probably, it should be the 4th sentence.

\section{Molecular identification of An. gambiae s.I}

Check and correct typo in line 1.

\section{Detection of LF14F ( Kdr allele)}

Correct typo on the title.

\section{Insecticide resistance status of An. gambiae s.I against different insecticides}

The study has revealed that, the only member of An. gambiae complex in this region is An. arabiensis. Therefore, i suggest the results should be changed to reflect this information correctly and report of the specific species.

\section{Avaliblity of LLINs}

Correct typo in the title.

\section{Table 6}

Check and correct typo in the title of the table.

\section{Discussion}

Check and correct typo in line 3.

In line 5, change the word "form" to be "from" for the sentence to have a proper meaning.

Check and correct typo in line 3 of paragraph 4. Is DDT still in use in Ethiopia? Authors may need to reflect its general use in Ethiopia.

\section{Conclusion And Recommendation}

Restructure sentence 2 to have a proper meaning.

The conclusion of high pyrethroid resistance in An. arabiensis should be shown in the results. In this study, the results indicated are for An. gambiae s.I., which is a wider group. 
